\title{
Stiripentol in Dravet syndrome: Results of a retrospective U.S. study
}

\author{
*Elaine C. Wirrell, †Linda Laux, †David N. Franz, §Joseph Sullivan, $₫$ Russell P. Saneto, \\ \#Richard P. Morse, **Orrin Devinsky, ††Harry Chugani, ††Angel Hernandez, $\S$ Lorie Hamiwka, \\ qףMohamad A. Mikati, \#\#Ignacio Valencia, ***Marie-Emmanuelle Le Guern, \\ *** Laurent Chancharme, and $+\dagger \dagger$ Marcio Sotero de Menezes
}

\begin{abstract}
*Divisions of Child and Adolescent Neurology, Department of Neurology, Mayo Clinic, Rochester, Minnesota, U.S.A.; $\uparrow$ Division of Neurology, Department of Pediatrics, Northwestern University Feinberg School of Medicine, Lurie Children's Hospital, Chicago, Illinois, U.S.A.; \$Departments of Pediatrics and Neurology, University of Cincinnati and Cincinnati Children’s Hospital Medical

Center, Cincinnati, Ohio, U.S.A.; §Division of Neurology, Department of Pediatrics, UCSF, San Francisco, California, U.S.A.; $\uparrow$ Division of Pediatric Neurology, University of Washington and Seattle Children's Hospital, Seattle, Washington, U.S.A.; \#Departments of Pediatrics and Neurology, Geisel School of Medicine, Dartmouth-Hitchcock Medical Center, Lebanon, New Hampshire, U.S.A.;

***Departments of Neurology, Neurosurgery and Psychiatry, NYU School of Medicine, New York, New York, U.S.A.;

$\dagger$ Children's Hospital of Michigan, Wayne State University, Detroit, Michigan, U.S.A.; \$ Division of Neurology, Cook

Children's Health Care System, Forth Worth, Texas, U.S.A.; §§Division of Neurology, Department of Pediatrics, Nationwide

Children's Hospital and the Ohio State University College of Medicine, Columbus, Ohio, U.S.A.; qqDivision of

Neurology, Department of Pediatrics, Duke University Medical Center, Durham, North Carolina, U.S.A.; \#Section of Neurology,

Departments of Pediatrics and Neurology, Drexel University College of Medicine, St Christopher's Hospital for Children, Philadelphia, Pennsylvania, U.S.A.; ***Biocodex Research Center, Compiegne, France; and

$\dagger \dagger \dagger$ Division of Neurology, Department of Pediatrics, Swedish Medical Center, Seattle, Washington,U.S.A.
\end{abstract}

\section{SUMMARY}

Purpose: To review the efficacy and tolerability of stiripentol in the treatment of U.S. children with Dravet syndrome.

Methods: U.S. clinicians who had prescribed stiripentol for two or more children with Dravet syndrome between March 2005 and 2012 were contacted to request participation in this retrospective study. Data collected included overall seizure frequency, frequency of prolonged seizures, and use of rescue medications and emergency room (ER)/hospital visits in the year preceding stiripentol initiation, and with stiripentol therapy. We separately assessed efficacy in the following treatment groups: group A, stiripentol without clobazam or valproate; group B, stiripentol with clobazam but without valproate; group $C$, stiripentol with valproate but without clobazam; and group D, stiripentol with clobazam and valproate. In addition, adverse effects were recorded.

Key Findings: Thirteen of 16 clinicians contacted for study participated and provided data on 82 children. Stiripentol was initiated a median of 6.0 years after seizure onset and I.2 years after diagnosis of Dravet syndrome. Compared to baseline, overall seizure frequency was reduced in $2 / 6$ in group A, 28/35 in group B, 8/14 in group C, and 30/48 in group D. All children with prolonged seizure frequency greater than quarterly during the baseline period experienced a reduction in this frequency on the various treatment arms with stiripentol. Similarly, 2/4 patients in group $A, 25 / 25$ in group $B, 5 / 10$ in group $C$, and $26 / 33$ in group $D$ experienced reduction in frequency of rescue medication use and $1 / 1$ in group $A, 12 / 12$ in group $B, 3 / 5$ in group $C$, and $18 / 19$ in group $D$ had reduction in frequency of ER/hospital visits. Adverse effects were reported in 38, most commonly sedation and reduced appetite. Four patients (5\%) discontinued stiripentol for adverse effects and two (2\%) for lack of efficacy.

Significance: Stiripentol is an effective and well-tolerated therapy that markedly reduced frequency of prolonged seizures in Dravet syndrome.

KEY WORDS: Stiripentol, Dravet syndrome, Pediatric epilepsy, Intractable epilepsy.
Dravet syndrome is a rare, catastrophic childhood epilepsy syndrome that is medically intractable and leads

Accepted June 3, 2013; Early View publication July 12, 2013.

Address correspondence to Elaine Wirrell, Division of Child and Adolescent Neurology, Mayo Clinic, 200 First St SW, Rochester, MN 55905, U.S.A.E-mail: wirrell.elaine@mayo.edu

Wiley Periodicals, Inc.

(C) 2013 International League Against Epilepsy to intellectual disability, motor impairment, and dependence in adulthood. In most cases, Dravet syndrome involves a de novo loss of function mutation in the SCN1A sodium channel gene. The estimated prevalence is approximately $1 / 40,900$ live births (Brunklaus et al., 2012), suggesting that 95-100 new cases of Dravet syndrome will be diagnosed in the United States each year. 


\section{E. C. Wirrell et al.}

Antiepileptic drugs are only partially effective for the treatment of Dravet syndrome-associated seizures (Chiron \& Dulac, 2011). Valproate and benzodiazepines (clobazam, clonazepam) are the most useful drugs, and open label studies have suggested that topiramate may also be effective (Coppola et al., 2002). In addition, both bromides and fenfluramine have been reported to be efficacious but are rarely used, with the latter being unavailable in the United States (Tanabe et al., 2008; Ceulemans et al., 2012). By contrast, carbamazepine and lamotrigine often exacerbate epilepsy and should generally be avoided. The ketogenic diet may also be effective, with two thirds to three fourths of children achieving a $>75 \%$ reduction in seizure frequency (Kang et al., 2005; Caraballo, 2011; Nabbout et al., 2011).

The efficacy of stiripentol in Dravet syndrome was initially confirmed in two small, randomized, placebo-controlled studies conducted in France and Italy (Chiron et al., 2000). Remarkable efficacy was noted when stiripentol was combined with valproate and clobazam, with approximately $30 \%$ of children on stiripentol versus none on placebo achieving freedom from generalized clonic or tonic-clonic seizures. In addition, in a study where patients were treated open-label with stiripentol for a median of 2.9 years, there was a significant and sustained reduction of seizure frequency and duration compared to baseline (Thanh et al., 2002). A multicenter study from Japan (Inoue et al., 2009) also provided additional evidence of unique efficacy in this medically intractable syndrome.

Because seizures can contribute to intellectual disability, and status epilepticus can result in numerous hospitalizations and death, stiripentol use may improve quality of life and long-term seizure and cognitive outcomes, decrease the number of emergency room (ER) visits, and possibly prolong survival for these children.

Stiripentol is registered as an orphan drug in Europe, Canada, and Japan. It is structurally unrelated to any currently marked antiepileptic agents, and it acts as an allosteric modulator of the $\gamma$-aminobutyric acid $(\mathrm{GABA})_{\mathrm{A}}$ receptor (Fisher, 2009). It displays anticonvulsant properties in a model of benzodiazepine-refractory status epilepticus in rodents (Grosenbaugh \& Mott, 2013). In addition, stiripentol inhibits the cytochrome P450 (CYP) system, resulting in increased concentrations of many antiepileptic drugs, most notably clobazam, by action on CYP2C19 (Tran et al., 1997).

Although not currently licensed in the United States, it may be obtained through special access, and some U.S. patients have received this therapy. However, given that the costs of non-U.S. Food and Drug Administration (FDA)approved drugs are generally not covered by insurers and that many neurologists will not prescribe a non-FDAapproved drug, most U.S. children with Dravet syndrome are unable to access this therapy. The present study was designed as a retrospective, multicenter survey of children with Dravet syndrome, treated with stiripentol at various
U.S. centers in order to evaluate the patterns of use of stiripentol, and under those conditions, its efficacy, side effects, and the nature of concurrent medications used.

\section{MeTHODS}

\section{Patient identification}

Orders for stiripentol from the United States received at Biocodex, marketing authorization holder for stiripentol, were reviewed and physicians who treated two or more children were contacted. The study goals and design were reviewed with each physician, and they were then invited to participate in this retrospective, noninterventional survey. Participating physicians were requested to provide data for all children clinically diagnosed with Dravet syndrome who were treated with stiripentol anytime in the 3 years prior to study onset.

\section{Data collected}

Each participating physician entered clinical data from their patient's charts onto standardized data collection forms in an anonymous manner. Information collected included demographic data (sex, age at seizure onset, and diagnosis of Dravet), presence of SCN1A mutation, neurologic examination findings, and developmental status both prior to stiripentol initiation and at last follow-up. Developmental status was defined as normal/borderline if the estimated or measured developmental quotient was 70 or higher, mildly delayed if 50 to $<70$, and moderately to severely delayed if $<50$.

Epilepsy details prior to stiripentol use and after its initiation including frequency of convulsive, absence, myoclonic and total seizures, prolonged seizures (defined as duration $>15 \mathrm{~min}$ ), rescue medication use (rectal diazepam, buccal diazepam or lorazepam, nasal midazolam or oral clonazepam), and emergency room visits or hospitalizations were collected. Overall seizure frequency was stratified into the following categories: (1) daily or more, (2) less than daily to weekly, (3) less than weekly to monthly, and (4) less than monthly to yearly. The frequency of convulsive seizures was stratified into the following: (1) greater than weekly, (2) less than or equal to weekly to monthly, (3) less than or equal to monthly to yearly, and (4) less than yearly or less. The frequency of prolonged seizures, rescue medication use, and emergency room/hospital visits were stratified into the following: (1) weekly or more, (2) less than weekly to monthly, (3) less than monthly to quarterly, and (4) less than quarterly.

Medical charts were reviewed to determine which of the following combinations each study participant had been treated with: (1) stiripentol without concurrent valproate or clobazam, (2) stiripentol with clobazam but without valproate, (3) stiripentol with valproate but without clobazam, and/or (4) stiripentol with concurrent clobazam and valproate. The majority of subjects received only 1-3 of 
these combinations. For each specific combination received by each participant, age at treatment and duration of treatment, overall seizure frequency and frequency of convulsive seizures, prolonged seizures, and rescue medication use on that combination was noted. No information about plasma levels was collected.

Data on other prophylactic antiepileptic drugs tried (noting reasons for discontinuation), use of dietary options (traditional ketogenic diet, modified Atkins diet, low glycemic index diet), vagus nerve stimulation, and epilepsy surgery were collected, and timing of each therapy relevant to stiripentol was noted.

In addition, adverse effects that occurred on stiripentol were recorded and the treating physician was asked to grade their perceived change in the child and family's quality of life with the addition of stiripentol (divided into marked improvement, mild improvement, no change, mild deterioration, and marked deterioration). These standardized forms were then returned to Biocodex for data entry and analysis.

\section{Data analysis}

\section{Efficacy of stiripentol in various combinations}

The frequencies of generalized convulsive, absence, myoclonic and total seizures, as well as prolonged seizures, use of rescue medication, and emergency room/hospital visits during the one year period prior to starting stiripentol (baseline period) were compared to each of the following treatment arms with stiripentol that the child received [(1) stiripentol without concurrent valproate or clobazam, (2) stiripentol with clobazam, no valproate, (3) stiripentol with valproate, no clobazam or (4) stiripentol with concurrent clobazam and valproate)]. Although clobazam and valproate were given a prominent role, as they are the most commonly used cotherapies with stiripentol, all other concurrently used antiepileptic medications were duly noted.

When assessing efficacy of each treatment arm, duration of therapy was considered. The proportion of children who had reduction (marked and mild) and increases (marked and mild) of overall seizure frequency, absence or myoclonic seizure frequency, prolonged seizures, use of rescue medication, and emergency room/hospital visits was also determined. Reduction in frequency of the various outcomes could be assessed only if a subject's treatment duration was longer than their baseline frequency. For example, overall seizure frequency reduction in a subject with a baseline frequency of less than weekly to monthly could be determined only if they were treated with the therapy for $>1$ month. Similarly, reduction in frequency of rescue medication in a patient with a baseline frequency of less than monthly to quarterly could be assessed only if they were treated for longer than 3 months. Marked reduction was defined as a decrease in frequency by at least two levels: "daily or more" to "at least less than weekly," "weekly or more" to "at least less than monthly," or "less than weekly to monthly" to "less than quarterly." Mild reduction was defined as a decrease in frequency of one level: "daily or more" to "at least less than daily to weekly" or "weekly or more" to "at least less than weekly to monthly," or "less than monthly to quarterly" to "less than quarterly." Marked increase was defined as a rise in frequency of at least two levels and mild increase was defined as a rise in frequency of one level.

\section{Tolerability of stiripentol}

Side effects were classified as minor if they did not result in discontinuation of stiripentol, and major if stiripentol was stopped as a result. The proportion of children developing mild and severe side effects, and the nature of side effects was reported.

All participating sites received approval from their respective institutional review boards.

\section{RESULTS}

Among the 16 physicians contacted who had prescribed stiripentol for Dravet syndrome in the preceding 3 years, 13 agreed to participate in the study. One physician did not respond to requests to join the study, one had relocated and no longer had access to medical records of stiripentol-treated patients, and the third was unable to get institutional review board approval for the study within the specified time limit. In total, 82 children with Dravet syndrome who received stiripentol through the special access program between March 2005 and 2012 were identified. The median number of subjects treated by participating physicians was 4 (25th percentile and 75th percentile, 2 and 10).

Demographic and clinical data are shown in Table 1. Stiripentol was initiated after a median of 6.0 years (25th percentile and 75th percentile, 3.8 and 9.3) following seizure onset and 1.2 years (25th percentile and 75 th percentile, 0.3 and 2.9) after diagnosis of Dravet syndrome.

At final follow-up, 44 children (54\%) were rated as having moderate to severe delay, 31 (38\%) as mild delay, and 6 (7\%) as normal/borderline (data was missing for one patient). Regarding ambulation, 73 (89\%) were independent ambulators without aides, $4(5 \%)$ were independent ambulators with aides, and $5(6 \%)$ were nonambulatory. Fifty-six $(68 \%)$ were reported to have behavioral problems.

\section{Efficacy of stiripentol}

Table 2 shows the number of subjects treated with each antiepileptic drug treatment arm, the median age at onset and duration of each treatment, and the frequency of generalized tonic-clonic seizures, all seizures, prolonged seizures, rescue medication use, and emergency room visits/hospitalizations for children prior to and after initiation of stiripentol. Only a small number of children were treated with stiripentol without concurrent clobazam and/or valproate, thereby limiting analysis in this subgroup. In 


\section{E. C. Wirrell et al.}

\begin{tabular}{|c|c|}
\hline \multicolumn{2}{|l|}{ Variable } \\
\hline Sex (male) & $38(46 \%)$ \\
\hline Median age at first seizure & 6.0 months ( 25 th and 75 th percentile, 4.0 and 8.0 ) \\
\hline Median age at diagnosis of Dravet syndrome & 5.4 years ( 25 th and 75 th percentile, 2.4 and 8.8 ) \\
\hline Median age at stiripentol initiation & 6.9 years ( 25 th and 75 th percentile, 4.2 and 10.4 ) \\
\hline \multicolumn{2}{|l|}{ Duration of stiripentol therapy in months } \\
\hline Median (25th and 75 th percentile ) & 22.3 months ( 9.9 and 43.2 ) \\
\hline Mean (standard deviation) & $28.5(20.3)$ \\
\hline SCNIA mutation present & $90.2 \%$ \\
\hline Median number of antiepileptic drugs tried prior to stiripentol initiation & 7.0 (25th and 75 th percentile, 6.0 and 9.0 ) \\
\hline Number with vagus nerve stimulator placed prior to stiripentol & $21(25.6 \%)$ \\
\hline $\begin{array}{l}\text { Number treated with dietary therapy (ketogenic diet, modified Atkins diet, } \\
\text { low glycemic diet) prior to stiripentol }\end{array}$ & $46(56.1 \%)$ \\
\hline Number undergoing epilepsy surgery prior to stiripentol & $6(7.3 \%)$ \\
\hline \multicolumn{2}{|l|}{ Developmental status prior to stiripentol ${ }^{a}(\%)$} \\
\hline Normal/borderline delay & II (I3.6) \\
\hline Mild delay & $24(29.6)$ \\
\hline Moderate/severe delay & $46(57.0)$ \\
\hline Median initial target dose of stiripentol in $\mathrm{mg} / \mathrm{kg} /$ day & 28 ( 25 th and 75 th percentile, 20 and 43 ) \\
\hline Median maximal dose of stiripentol over treatment course in $\mathrm{mg} / \mathrm{kg} / \mathrm{day}$ & 42 ( 25 th and 75 th percentile, 30 and 56 ) \\
\hline Median stiripentol dose at last follow-up in $\mathrm{mg} / \mathrm{kg} / \mathrm{day}$ & 30 (25th and 75 th percentile, 22 and 43 ) \\
\hline
\end{tabular}

addition, the median duration of treatment with stiripentol with valproate but without clobazam was only 3.1 months.

Compared to the baseline period, most children who received stiripentol in the various treatment arms showed reductions in overall seizure frequency and in frequency of both use of rescue medications and emergency room/hospital visits.

Table 3 shows the change from baseline period in overall seizure frequency, as well as prolonged seizures, frequency of rescue medication use, and frequency of emergency room/hospital visits.

\section{Stiripentol without clobazam or valproate}

Six children were treated with stiripentol without concurrent clobazam or valproate, and median treatment duration was 6.3 months (25th and 75th percentile, 3.1 and 8.0). Children received a median of two other antiepileptic drugs during this time (25th and 75th percentile, 1 and 3), consisting of clonazepam (3/6), topiramate (3/6), levetiracetam $(2 / 6)$, felbamate (1/6), primidone $1 / 6)$, and tiagabine (1/6).

Although the numbers are small, overall seizure frequency with stiripentol was reduced in two, unchanged in three, and increased in one compared to the baseline period. Both prolonged seizure frequency and emergency room/ hospital visit frequency were markedly reduced in the only patient experiencing them at least quarterly during the baseline period. Of the four patients using rescue medication more than quarterly during the baseline period, two showed reduction in use (one marked, one mild), one increased use (mild), and in one child use was unchanged. Conversely, one child with prolonged seizure frequency less than quarterly during the baseline period experienced a marked increase to less than weekly to monthly with stiripentol. However, no patient with frequency of rescue medication use or emergency room/hospital visits less than quarterly had worsening in these parameters with stiripentol.

None of three children who had absences more than monthly during the baseline period experienced significant change in that frequency. Of three children with myoclonic seizures greater than monthly at baseline, only one showed mild improvement and in two myoclonic frequency was unchanged.

\section{Stiripentol with clobazam but without valproate}

Thirty-five children received stiripentol with clobazam but without concurrent valproate for a median treatment duration of 21.8 months (25th and 75th percentile, 9.5 and 43.9). Children received a median of one other antiepileptic drug during this time (25th and 75th percentile, 1 and 2) consisting of topiramate (19/35), levetiracetam (7/35), felbamate $(5 / 35)$, ethosuximide $(4 / 35)$, zonisamide $(3 / 35)$, phenobarbital (3/35), clonazepam (2/35), lamotrigine (2/35), methsuximide (1/35), and steroids (1/35). In addition, one child received nutritional supplements and another received verapamil.

Compared to the baseline period, overall seizure frequency was reduced in $28(80 \%)$ (markedly in 11 and mildly in 17), unchanged in $7(20 \%)$, and increased in none. Frequencies of prolonged seizures, rescue medication use and emergency room/hospital visits were reduced 


\begin{tabular}{|c|c|c|c|c|c|}
\hline & $\begin{array}{l}\text { Year prior to } \\
\text { stiripentol } \\
\text { initiation }\end{array}$ & $\begin{array}{l}\text { Stiripentol } \\
\text { without } \\
\text { valproate or } \\
\text { clobazam }\end{array}$ & $\begin{array}{c}\text { Stiripentol with } \\
\text { clobazam but } \\
\text { without } \\
\text { valproate }\end{array}$ & $\begin{array}{l}\text { Stiripentol with } \\
\text { valproate but } \\
\text { without } \\
\text { clobazam }\end{array}$ & $\begin{array}{l}\text { Stiripentol with } \\
\text { clobazam and } \\
\text { valproate }\end{array}$ \\
\hline Number of patients treated & 82 & 6 & 35 & 15 & 48 \\
\hline Median age at treatment onset in months & $\begin{array}{c}6.9 \text { (age at } \\
\text { STP initiation) }\end{array}$ & 6.1 & 6.1 & 5.4 & 8.6 \\
\hline $\begin{array}{l}\text { Median duration of treatment in months } \\
\text { ( } 25 \text { th and } 75 \text { th percentile) }\end{array}$ & $12(12,12)$ & $6.3(3.1,8.0)$ & $21.8(9.5,43.9)$ & $3.1(0.9,12.8)$ & $20.7(10.5,35.5)$ \\
\hline \multicolumn{6}{|l|}{ Frequency of generalized convulsive seizures (\%) } \\
\hline Yearly or less & $7(9)$ & $2(33)$ & $10(29)$ & $3(20)$ & $18(37)$ \\
\hline$\leq$ Monthly to >yearly & $10(12)$ & $\mathrm{I}(\mathrm{I7})$ & $8(23)$ & $4(27)$ & $8(17)$ \\
\hline$\leq$ Weekly to >monthly & $27(33)$ & $\mathrm{I}(\mathrm{I7})$ & $13(37)$ & $7(47)$ & $12(25)$ \\
\hline >Weekly & $38(46)$ & $2(33)$ & $4(11)$ & $\mathrm{I}(7)$ & $10(21)$ \\
\hline \multicolumn{6}{|l|}{ Frequency of all seizures combined (\%) } \\
\hline$<$ Monthly to yearly & 0 & 0 & $10(29)$ & $2(13)$ & $17(35)$ \\
\hline$<$ Weekly to monthly & $18(22)$ & $2(33)$ & $16(46)$ & $6(40)$ & $9(19)$ \\
\hline$<$ Daily to weekly & $23(28)$ & $3(50)$ & $6(17)$ & $4(27)$ & II (23) \\
\hline Daily or more & $4 \mid(50)$ & $\mathrm{I}(\mathrm{I7})$ & $3(9)$ & $3(20)$ & II (23) \\
\hline Frequency of prolonged seizures (\%) & 3 data missing & & I data missing & & \\
\hline$<$ Quarterly & $51(65)$ & $5(83)$ & $32(94)$ & $9(60)$ & $45(94)$ \\
\hline$<$ Monthly to quarterly & $6(8)$ & 0 & $2(6)$ & $2(13)$ & $\mathrm{I}(2)$ \\
\hline$<$ Weekly to monthly & $16(20)$ & $\mathrm{I}(\mathrm{I7})$ & 0 & $4(27)$ & 0 \\
\hline Weekly or more & $6(8)$ & 0 & 0 & 0 & $2(4)$ \\
\hline \multicolumn{6}{|l|}{ Status epilepticus ( $\geq 30 \mathrm{~min})$} \\
\hline Yes $(\mathrm{N}, \%)$ & $44(54)$ & $0(0)$ & I (3) & $3(20)$ & $5(10)$ \\
\hline Mean duration of longest status in minutes & 186 & & 60 & 105 & 201 \\
\hline Frequency of rescue medication use (\%) & 5 data missing & & & & \\
\hline$<$ Quarterly & $17(22)$ & $2(33)$ & $23(66)$ & $3(20)$ & $33(69)$ \\
\hline$<$ Monthly to quarterly & $16(21)$ & $2(33)$ & $6(17)$ & $2(13)$ & $4(8)$ \\
\hline$<$ Weekly to monthly & $23(30)$ & $2(33)$ & $6(17)$ & $9(60)$ & $5(10)$ \\
\hline Weekly or more & $21(27)$ & 0 & 0 & I (7) & $6(13)$ \\
\hline Frequency of ER visits or hospitalizations (\%) & 3 data missing & & I data missing & & 2 data missing \\
\hline$<$ Quarterly & $51(65)$ & $6(100)$ & $32(94)$ & $7(47)$ & $42(91)$ \\
\hline$<$ Monthly to quarterly & $20(25)$ & 0 & $2(6)$ & $4(27)$ & $3(7)$ \\
\hline$<$ Weekly to monthly & $6(8)$ & 0 & 0 & $3(20)$ & 0 \\
\hline Weekly or more & $2(3)$ & 0 & 0 & $I(7)$ & $I(2)$ \\
\hline
\end{tabular}

in all children who had experienced these outcomes at least quarterly during the baseline period, and the degree of reduction was marked in $82 \%, 52 \%$, and $83 \%$ of cases, respectively. One of 22 children who had prolonged seizures less than quarterly and another 1/22 who had emergency room/hospital visits less than quarterly during the baseline period experienced a mild increase with stiripentol/clobazam to less than monthly to quarterly. In addition, one of eight children using rescue medication less than quarterly during the baseline period experienced a mild increase in use to less than monthly to quarterly after stiripentol/clobazam was added.

Of 14 with absence seizures more than monthly during baseline, 10 showed reduction (five marked and five mild), whereas four had no change in frequency. Of 11 with myoclonic seizures greater than monthly at baseline, eight showed reduction (five marked and three mild), and in three frequency was unchanged.

\section{Stiripentol with valproate but without clobazam}

Fifteen children received stiripentol and valproate without concurrent clobazam for a median duration of 3.1 months (25th and 75th percentile, 0.9 and 12.8). Children received a median of one other antiepileptic drug during this time (25th and 75th percentile, 1 and 2) including clonazepam (6/15), topiramate (4/15), clorazepate (4/15), levetiracetam (4/15), ethosuximide (2/15), zonisamide (2/ 15), phenobarbital (1/15), lorazepam (1/15), and acetazolamide (1/15).

Overall seizure frequency was reduced in 8/14 (57\%) (four marked and four mild), unchanged in 6 (43\%), and increased in none compared to the baseline period. Prolonged seizure frequency was reduced in all children $(80 \%$ markedly) experiencing these at least quarterly during the baseline period. Frequency of rescue medication use was reduced in $5(50 \%)$, unchanged in $3(30 \%)$, and increased in $2(20 \%)$ of those with at least quarterly use during the 


\section{E. C. Wirrell et al.}

Table 3. Change from baseline in overall seizure frequency and in those having prolonged seizures, use of rescue medication or ER/hospital admission at least quarterly

\begin{tabular}{|c|c|c|c|c|}
\hline & $\begin{array}{l}\text { Overall seizure } \\
\text { frequency } \\
N(\%)\end{array}$ & $\begin{array}{c}\text { Prolonged seizure } \\
\text { frequency } \\
\mathrm{N}(\%)\end{array}$ & $\begin{array}{c}\text { Frequency of rescue } \\
\text { medication use } \\
N(\%)\end{array}$ & $\begin{array}{c}\text { Frequency of ER } \\
\text { visits or hospitalizations } \\
\mathrm{N}(\%)\end{array}$ \\
\hline \multicolumn{5}{|c|}{ STP without clobazam or valproate } \\
\hline $\mathrm{N}$ & 6 & I & 4 & 1 \\
\hline Reduction & $2(33)$ & $I(100)$ & $2(33)$ & I $(100)$ \\
\hline Marked & 0 & $I(100)$ & $\mathrm{I}(17)$ & I $(100)$ \\
\hline Mild & $2(33)$ & 0 & $\mathrm{I}(\mathrm{I} 7)$ & 0 \\
\hline Unchanged & $3(50)$ & 0 & $\mathrm{I}(\mathrm{I})$ & 0 \\
\hline Increase & $\mathrm{I}(17)$ & 0 & I (I7) & 0 \\
\hline Marked & 0 & 0 & 0 & 0 \\
\hline Mild & $\mathrm{I}(\mathrm{I7})$ & 0 & $I(I 7)$ & 0 \\
\hline \multicolumn{5}{|c|}{ STP plus clobazam } \\
\hline $\mathrm{N}$ & 35 & $11^{a}$ & $25^{b}$ & $12^{c}$ \\
\hline Reduction & $28(80)$ & II (I00) & $25(100)$ & $12(100)$ \\
\hline Marked & II (3I) & $9(82)$ & $13(52)$ & $10(83)$ \\
\hline Mild & $17(49)$ & $2(18)$ & $12(48)$ & $2(17)$ \\
\hline Unchanged & $7(20)$ & 0 & 0 & 0 \\
\hline Increase & 0 & 0 & 0 & 0 \\
\hline Marked & 0 & 0 & 0 & 0 \\
\hline Mild & 0 & 0 & 0 & 0 \\
\hline \multicolumn{5}{|c|}{ STP plus valproate } \\
\hline$N$ & $14^{\mathrm{d}}$ & $5^{d}$ & $10^{e}$ & $5^{f}$ \\
\hline Reduction & $8(57)$ & $5(100)$ & $5(50)$ & $3(60)$ \\
\hline Marked & $4(29)$ & $4(80)$ & $I(10)$ & $2(40)$ \\
\hline Mild & $4(29)$ & I (20) & $4(40)$ & I (20) \\
\hline Unchanged & $6(43)$ & 0 & $3(30)$ & $2(40)$ \\
\hline Increase & 0 & 0 & $2(20)$ & 0 \\
\hline Marked & 0 & 0 & 0 & 0 \\
\hline Mild & 0 & 0 & $2(20)$ & 0 \\
\hline \multicolumn{5}{|c|}{ STP plus clobazam and valproate } \\
\hline$N$ & 48 & $18^{a}$ & $33^{g}$ & $19^{h}$ \\
\hline Reduction & $30(63)$ & $18(100)$ & $26(79)$ & $18(95)$ \\
\hline Marked & $17(35)$ & $16(89)$ & $16(48)$ & II (58) \\
\hline Mild & $13(27)$ & $2(I I)$ & $10(30)$ & $7(37)$ \\
\hline Unchanged & $16(33)$ & 0 & $4(12)$ & $\mathrm{I}(5)$ \\
\hline Increase & $2(4)$ & 0 & $3(9)$ & 0 \\
\hline Marked & 0 & 0 & I (3) & 0 \\
\hline Mild & $2(4)$ & 0 & $2(6)$ & 0 \\
\hline \multicolumn{5}{|c|}{ 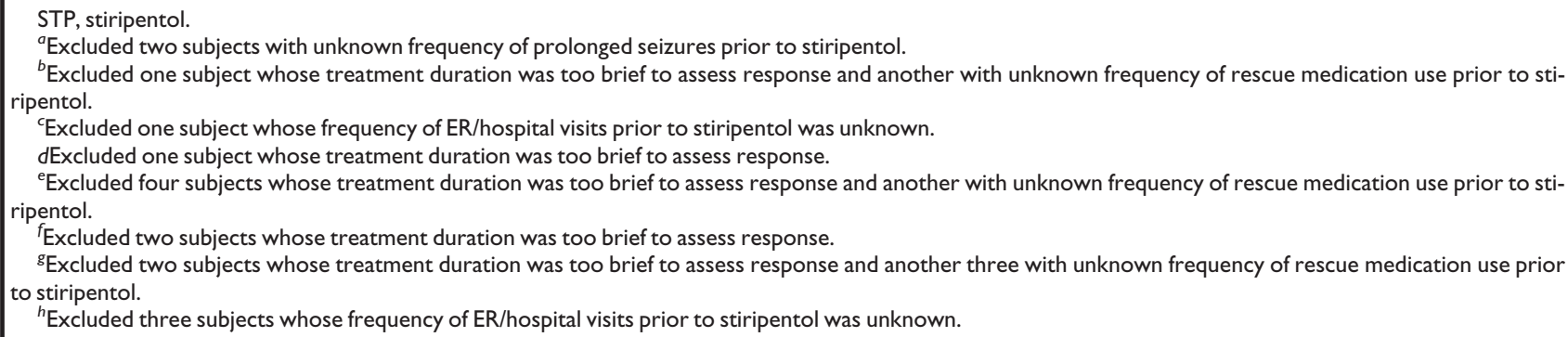 } \\
\hline
\end{tabular}

baseline period. The frequency of emergency room/hospital visits was reduced in $3(60 \%)$ and unchanged in $2(40 \%)$ of those with visits at least quarterly during the baseline period. No child who had prolonged seizures, rescue medication use, or emergency room/hospital visits less than quarterly during the baseline period experienced an increase in these frequencies with stiripentol/valproate therapy.

Of seven children with absence seizures more than monthly at baseline, two showed reduction (one marked and one mild) and frequency was unchanged in the remaining five. Of 10 with myoclonic seizures greater than monthly at baseline, 7 showed reduction ( 3 marked and 4 mild) and frequency was unchanged in the remaining 3 .

\section{Stiripentol with concurrent clobazam and valproate}

Forty-eight children received stiripentol with concurrent valproate and clobazam for a median duration of 20.7 months (25th and 75th percentile, 10.5 and 35.5). 
Children received a median of 1 other antiepileptic drug during this time (25th and 75th percentile, 0 and 2), which consisted of topiramate (14/48), levetiracetam (9/48), ethosuximide (3/48), clonazepam (2/48), clorazepate (2/48), phenobarbital (2/48), phenytoin (2/48), lamotrigine (2/48), felbamate (1/48), vigabatrin $(1 / 48)$, zonisamide (1/48), lorazepam (1/48), methsuximide (1/48), and intravenous gammaglobulin (1/48). One child also received verapamil.

Overall seizure frequency was reduced in 30 (63\%) (17 marked and 13 mild), unchanged in $16(33 \%)$, and increased in $2(4 \%)$ (both mild) compared to the baseline period. Prolonged seizure frequency was reduced in all 18 children (16 marked and 2 mild) who had prolonged seizures at least quarterly during the baseline period. Frequency of rescue medication use was reduced in $26(79 \%)$, unchanged in 4 (12\%), and increased in 3 (9\%) (1 marked and 2 mild) of 33 children who used this at least quarterly during the baseline period. The frequency of emergency room/hospital visits was reduced in 18/19 (95\%) and unchanged in $1(5 \%)$ of those with visits at least quarterly during the baseline period. Only $2 / 28$ children with a prolonged seizure frequency of less than quarterly, 1/10 with rescue medication use less than quarterly, and 1/26 with emergency room/hospital visit frequency less than quarterly during the baseline period had an increase in these frequencies with stiripentol/clobazam/ valproate.

Of 19 with absence seizures more than monthly at baseline, 13 showed reduction ( 8 marked and 5 mild), 5 were unchanged, and 1 had a mild increase in absence frequency. Of 21 with myoclonic seizures more than monthly at baseline, 14 showed reduction (7 marked and 7 mild), and 7 had no change in frequency.

\section{Tolerability}

The median maximal dose of stiripentol used in the present study was $42 \mathrm{mg} / \mathrm{kg} / \mathrm{day}$ (25th and 75th percentile, 30 and 56), and the median dose at last follow-up was $30 \mathrm{mg} /$ $\mathrm{kg} / \mathrm{day}$ (25th and 75th percentile, 22 and 43).

Adverse effects were reported in 31 patients (38\%), being mild and not requiring stiripentol discontinuation in the majority. There was no significant difference in maximal doses of stiripentol in patients with and without adverse effects ( 43.5 vs. $46.1 \mathrm{mg} / \mathrm{kg} / \mathrm{day})$. Table 4 illustrates which adverse effects were reported and their frequencies. The most common adverse effects were sedation and decrease in appetite. Some episodes of "seizure" or "convulsions" were reported as adverse events by several investigators. However, as all subjects had seizures, we considered these as part of the disease, and reflective of poor efficacy rather than truly an adverse event. Only four (5\%) discontinued stiripentol for adverse effects (two reduced appetite/weight loss and two worsening behavior). In addition, two children stopped for lack of efficacy and one for financial reasons.

Over the study period, two children (2.4\%) died while taking stiripentol; however, the causes of death were judged
Table 4. Adverse effects reported with stiripentol

\begin{tabular}{|lc|}
\hline & $\begin{array}{c}\text { Number }(\%) \text { of } \\
\text { patients }(\mathrm{N}=82)\end{array}$ \\
\hline Nervous system signs and symptoms & $22(26.8)$ \\
Sedation/somnolence & $15(18.3)$ \\
Ataxia or balance disorder & $4(4.9)$ \\
Seizures/convulsions & $3(3.7)$ \\
Drooling & $\mathrm{I}(\mathrm{I} .2)$ \\
Gait apraxia & $\mathrm{I}(\mathrm{I} .2)$ \\
Psychomotor hyperactivity & $\mathrm{I}(\mathrm{I} .2)$ \\
Slow speech & $\mathrm{I}(\mathrm{I} .2)$ \\
Tremor & $\mathrm{I}(\mathrm{I} .2)$ \\
Psychiatric/behavioral signs and symptoms & $6(7.3)$ \\
Insomnia & $3(3.7)$ \\
Abnormal behavior or aggression & $2(2.4$ \\
Depressed mood & $\mathrm{I}(\mathrm{I} .2)$ \\
Stuttering & $\mathrm{I}(\mathrm{I} .2)$ \\
Hallucination, visual & $\mathrm{I}(\mathrm{I} .2)$ \\
General signs and symptoms & $8(9.8)$ \\
Fatigue & $2(2.4)$ \\
Crying or irritability & $2(2.4)$ \\
Pain & $\mathrm{I}(\mathrm{I} .2)$ \\
Weight loss & $4(4.9)$ \\
Eye signs and symptoms & $\mathrm{I}(\mathrm{I} .2)$ \\
Scleral icterus ${ }^{a}$ & $\mathrm{I}(\mathrm{I} .2)$ \\
Gastrointestinal signs and symptoms & $8(9.8)$ \\
Vomiting & $\mathrm{I}(\mathrm{I} .2)$ \\
Decreased appetite & $7(8.5)$ \\
Liver function test abnormal & $\mathrm{I}(\mathrm{I} .2)$ \\
Skin and subcutaneous tissue signs and symptoms & $\mathrm{I}(\mathrm{I} .2)$ \\
Photosensitivity reaction & $\mathrm{I}(\mathrm{I} .2)$ \\
\hline${ }^{a}$ Patient also had abnormal liver function tests. & \\
\hline
\end{tabular}

by the prescribing physician to be unrelated to stiripentol. The annual mortality rate was $1.02 \%$, based on the total treatment duration of our subjects of 194.75 patient years. A 4-year-old girl, who had been on stiripentol for 4 months, was found dead the day following a tonsillectomy. No autopsy was performed. In the second case, a 6-year-old girl, who had been on stiripentol for 3 years in conjunction with valproate and clobazam, was found dead in the morning, face down on a pillow. No seizure had been witnessed and an autopsy was consistent with sudden unexplained death in epilepsy, showing an anatomically normal brain with no other anatomic cause of death discernible.

Based on the treating physician's impression, patient and family quality of life was markedly improved in 40 (49\%), somewhat improved in $32(39 \%)$, unchanged in $4(5 \%)$, mildly deteriorated in $3(4 \%)$, and markedly deteriorated in $3(4 \%)$.

\section{Discussion}

The cohort of children in this study had the typical clinical features of Dravet syndrome with severe epilepsy, frequent seizures of varying semiologies, recurrent status epilepticus which often led to hospital admission, and medical intractability. The majority experienced reduction 
in frequency of prolonged seizures, rescue medication use, and emergency room/hospital visits with stiripentol. In addition, most children showed mild or marked reduction in overall seizure frequency, as well as absence and myoclonic seizure frequency. This finding is notable, since these patients had documented intractability, having tried a median of seven antiepileptic drugs that failed to adequately control seizures. Over half had also tried dietary therapy without efficacy, and many failed vagus nerve stimulation therapy. Notably, stiripentol dose used in this study was often lower than the recommended dosage of $50 \mathrm{mg} / \mathrm{kg} /$ day.

The efficacy of stiripentol for seizure control in our cohort is consistent with previous work. In a double-blind, randomized, placebo-controlled trial of stiripentol added on to both clobazam and valproate, $71 \%$ showed a $>50 \%$ reduction in tonic-clonic seizure frequency during the second month of stiripentol treatment (Chiron et al., 2000). Further studies have confirmed the unique efficacy of stiripentol in Dravet syndrome (Thanh et al., 2002; Inoue et al., 2009).

Stiripentol acts by various mechanisms. It significantly increases the GABAergic postsynaptic currents at clinically relevant concentrations and in a concentration-dependent manner, by enhancing the opening duration as opposed to the opening frequency of the chloride channel (Quilichini et al., 2006). Stiripentol is a direct allosteric modulator of the $\mathrm{GABA}_{\mathrm{A}}$ receptor, with particular affinity for the $\alpha 3$-containing receptors, a type of subunit highly expressed in the developing brain (Laurie et al., 1992; Fisher, 2009).

In addition, stiripentol is a potent inhibitor of the cytochrome P450 system, with particular inhibition of CYP2C19 and additional inhibition of CYP1A2, CYP2C9, and CYP3A4. Stiripentol is most commonly combined with valproate and clobazam in treating Dravet syndrome, resulting in no appreciable change in plasma valproate concentrations but approximately twofold increases in clobazam and five- to sevenfold increases in norclobazam levels (Giraud et al., 2006). Therefore, efficacy may result in part from elevated clobazam and norclobazam levels. We found support for a synergistic effect of stiripentol and clobazam, as seizure control on this combination (with or without valproate) appeared better than without clobazam cotherapy. However, although the number of children treated without clobazam cotherapy was relatively small in this study, our results suggest independent benefit from stiripentol alone. Frequency of prolonged seizures was reduced, often markedly, and most patients also had reductions in the use of rescue medication and emergency room/hospital visits. Our results are similar to those of the open-label Japanese study, in which short-term responder rates of $61 \%$ were seen, even though only half of children received clobazam cotherapy (Inoue et al., 2009). These clinical results are consistent with the patch-clamp recording findings by Fisher that stiripentol acts directly on the $\mathrm{GABA}_{\mathrm{A}}$ receptors at a site distinct from benzodiazepines, that both stiripentol and clobazam/nor- clobazam act independently at $\mathrm{GABA}_{\mathrm{A}}$ receptors, and that the combination of their effects increases the maximum response beyond that of either drug alone (Fisher, 2011).

Somewhat surprisingly, we found that children treated with valproate, clobazam, and stiripentol did not have better seizure control than those treated with stiripentol and clobazam without valproate. Whether valproate provides further benefit in subjects already receiving clobazam and stiripentol will need to be evaluated in a prospective study.

Very few subjects were reported to have increased seizures. A mild increase in seizure frequency was reported in only $1 / 6$ children on stiripentol without clobazam or valproate, and 2/48 children treated with stiripentol with clobazam and valproate. Only $2 \%$ discontinued stiripentol for lack of efficacy, which is notable given that a median of seven antiepileptic therapies had previously failed to adequately control seizures.

Stiripentol was well tolerated. Similar to results of previous studies, the most common adverse effects were sedation, decreased appetite with poor weight gain, insomnia, and ataxia. Many adverse effects were effectively managed by dose reduction of stiripentol and/or comedications. Only $5 \%$ discontinued stiripentol due to adverse effects, which included reduced appetite/weight loss in two and worsening behavior in two. Two subjects died during stiripentol treatment but deaths were considered unrelated in both cases.

Dravet syndrome carries a significant risk of other comorbidities. Similar to our results, Genton et al. (2011) reported problematic long-term outcomes, with persisting seizures, cerebellar dysfunction, gait impairment, moderate to severe intellectual disability, and behavior concerns based on their series of 24 patients followed into adulthood. Although these comorbidities are likely not solely the result of poor seizure control, prevention of prolonged seizures, reduction of overall seizure frequency will likely improve long-term outcome for patients with Dravet syndrome. Furthermore, many of our subjects also showed reductions in absence and myoclonic seizures, which have been reported to be associated with greater risk of cognitive impairment (Ragona, 2011).

Mortality is elevated in Dravet syndrome, with studies reporting that $7-21 \%$ of children die before reaching adulthood (Akiyama et al., 2010; Genton et al., 2011; Sakauchi et al., 2011; Skluzacek et al., 2011). Status epilepticus and its consequences account for a large number of deaths in younger patients, and sudden unexplained death in epilepsy results in deaths at all ages. Based on their data from the International Dravet Syndrome Epilepsy Action League patient registry, Skluzacek et al. (2011) reported an annual mortality rate from sudden unexplained death in epilepsy of $0-1.3 \%$ per year (mean $0.6 \%$ ) and from fatal status epilepticus of $0-1.32 \%$ per year (mean $0.37 \%$ ). In our study, neither the overall annual mortality rate of $1.02 \%$ nor the overall rate of $2.4 \%$ appears excessive.

Despite its efficacy in Dravet syndrome, stiripentol was used late in the course-only after a median of seven 
antiepileptic medications, and often dietary therapy, vagus nerve stimulation, and even epilepsy surgery had failed. In part, this is because of a delayed diagnosis of Dravet syndrome, as the median time from first seizure until definitive diagnosis was 4.8 years. However, even after definitive diagnosis, initiation of stiripentol was additionally delayed by a median of 1.2 years, likely the result of difficulty accessing a medication that is not currently approved for use in the United States, and the cost barrier for many families.

The present study had a number of limitations. Given its design, the overall seizure frequency, frequency of prolonged seizures, rescue medication use, and emergency room/hospital visits were recorded retrospectively based on chart review, which is less accurate than prospective counts. In addition, children were on a varied number and type of other antiepileptic therapies. We did not formally assess dose changes in those but presumed that stiripentol was being added because prior medications had failed to provide adequate seizure control. Given the prominent pharmacologic interaction with many antiepileptic medications such as clobazam, doses of these were often reduced when stiripentol was added. Although medications such as carbamazepine and lamotrigine have been reported to exacerbate seizures in Dravet syndrome, only a very small proportion of our cohort received these, and thus discontinuation of these medications could not explain the dramatic improvement in seizure control seen in so many of our subjects. The target dose of stiripentol varied between patients and the median dose was relatively low, but it still afforded good seizure control in many children. The subject number in certain treatment arms (particularly stiripentol without clobazam or valproate) was low, limiting our ability to draw conclusions on its efficacy. The low number in this subgroup likely reflects prior work that suggests stiripentol should be used as a cotherapy with valproate and/or clobazam (Chiron et al., 2000). The duration of treatment in certain arms (particularly stiripentol with valproate but without clobazam) was also brief, which likely limited our ability to document efficacy, particularly in those with low baseline frequencies. Adverse effects were based on chart review, which likely limited detection of milder symptoms. Furthermore, levels of comedications were not routinely obtained or recorded. Given that stiripentol has prominent pharmacokinetic interactions with many antiepileptic drugs, it is difficult to know whether reported adverse effects were due primarily to stiripentol or rather to altered concentrations of another comedication, resulting from addition of stiripentol. Finally, although we assessed frequency of rescue medication use, it is known that some families use rescue medication even for brief seizures. Therefore, reduction in frequency of use may either reflect decreased frequency of prolonged seizures, or decreased overall seizure frequency.

Given the present results and those of other studies (Chiron et al., 2000; Inoue et al., 2009), we believe that stiripentol should be considered earlier in the therapeutic armamentarium for Dravet syndrome, particularly if clobazam and valproate have failed to provide adequate seizure control and before trying topiramate, levetiracetam, ethosuximide, and other less proven drugs. This approach will also reduce exposure to ineffective antiepileptic drugs and their adverse effects, reduce health care costs for emergency room visits, hospitalizations, and rescue medication, and most importantly improve quality of life for children with Dravet syndrome and their families.

\section{Acknowledgments}

We wish to acknowledge the assistance of the following individuals: Diana Umanzor (Anne and Robert H. Lurie Children's Hospital of Chicago), Ida Foo (UCSF Epilepsy Center), Katherine Nickels MD and Lily Wong-Kisiel MD (Mayo Clinic), Laurie Guildry, BS RN (Seattle Children's Hospital), Aravindhan Veerapandiyan MB and Karen Cornett BS (Duke University), Jane Kwon (NYU), Juli Kidd RN, Noelia Ramirez, and Saleem I Malik MD (Cook Children's Health Care System), Kathleen Pawlik MSN RN CPNP (Children's Hospital of Michigan).

\section{Disclosure}

This study was supported by Biocodex. Each of the institutions received compensation for the participation of each investigator; however, investigators received no direct payment. Dr. Franz has received honoraria from Novartis and Lundbeck and receives research funding from Novartis. Dr. Saneto has received travel grants from Dravet.org and CURE. Dr. Devinsky has served as a paid consultant for UCB and Biocodex. Dr. Hernandez has received honoraria from Lundbeck and Cyberonics, and receives grant money from National Institute of Neurological Disorders and Stroke (NINDS). Dr. Mikati has received grant funding or honoraria from Cyberonics, GlaxoSmithKline, UCB, Pfizer, Cubist, Athena, and Lundbeck. Drs. Le Guern and Chancharme are paid employees of Biocodex. Drs. Wirrell, Morse, Chugani, Laux, Hamiwka, Valencia, Sullivan, and Sotero de Menezes have no other conflicts of interest to disclose. We confirm that we have read the Journal's position on issues involved in ethical publication and affirm that this report is consistent with those guidelines.

\section{REFERENCES}

Akiyama M, Kobayashi K, Yoshinaga H, Ohtsuka Y. (2010) A long-term follow-up study of Dravet syndrome up to adulthood. Epilepsia 51:1043-1052.

Brunklaus A, Ellis R, Reavey E, Forbes GH, Zuberi SM. (2012) Prognostic, clinical and demographic features in SCN1A mutation-positive Dravet syndrome. Brain 135:2329-2336.

Caraballo RH. (2011) Nonpharmacologic treatments of Dravet syndrome: focus on the ketogenic diet. Epilepsia 52(Suppl. 2):79-82.

Ceulemans B, Boel M, Leyssens K, Van Rossem C, Neels P, Jorens PG, Lagae L. (2012) Successful use of fenfluramine as an add-on treatment for Dravet syndrome. Epilepsia 53:1131-1139.

Chiron C, Dulac O. (2011) The pharmacologic treatment of Dravet syndrome. Epilepsia 52(Suppl. 2):72-75.

Chiron C, Marchand MC, Tran A, Rey E, d'Athis P, Vincent J, Dulac O, Pons G. (2000) Stiripentol in severe myoclonic epilepsy in infancy: a randomised placebo-controlled syndrome-dedicated trial. STICLO study group. Lancet 356:1638-1642.

Coppola G, Capovilla G, Montagnini A, Romeo A, Spano M, Tortorella G, Veggiotti P, Viri M, Pascotto A. (2002) Topiramate as add-on drug in severe myoclonic epilepsy in infancy: an Italian multicenter open trial. Epilepsy Res 49:45-48.

Fisher JL. (2009) The anti-convulsant stiripentol acts directly on the GABA (A) receptor as a positive allosteric modulator. Neuropharmacology 56:190-197. 


\section{E. C. Wirrell et al.}

Fisher JL. (2011) Interactions between modulators of the GABA(A) receptor: stiripentol and benzodiazepines. Eur J Pharmacol 654:160-165.

Genton P, Velizarova R, Dravet C. (2011) Dravet syndrome: the long-term outcome. Epilepsia 52(Suppl. 2):44-49.

Giraud C, Treluyer JM, Rey E, Chiron C, Vincent J, Pons G, Tran A. (2006) In vitro and in vivo inhibitory effect of stiripentol on clobazam metabolism. Drug Metab Dispos 34:608-611.

Grosenbaugh DK, Mott DD. (2013) Stiripentol is anticonvulsant by potentiating GABAergic transmission in a model of benzodiazepinerefractory status epilepticus. Neuropharmacology 67:136-143.

Inoue Y, Ohtsuka Y, Oguni H, Tohyama J, Baba H, Fukushima K, Ohtani H, Takahashi Y, Ikeda S. (2009) Stiripentol open study in Japanese patients with Dravet syndrome. Epilepsia 50:2362-2368.

Kang HC, Kim YJ, Kim DW, Kim HD. (2005) Efficacy and safety of the ketogenic diet for intractable childhood epilepsy: Korean multicentric experience. Epilepsia 46:272-279.

Laurie DJ, Wisden W, Seeburg PH. (1992) The distribution of thirteen GABAA receptor subunit mRNAs in the rat brain. III. Embryonic and postnatal development. J Neurosci 12:4151-4172.

Nabbout R, Copioli C, Chipaux M, Chemaly N, Desguerre I, Dulac O, Chiron C. (2011) Ketogenic diet also benefits Dravet syndrome patients receiving stiripentol: a prospective pilot study. Epilepsia 52:e54-e57.

Quilichini PP, Chiron C, Ben-Ari Y, Gozlan H. (2006) Stiripentol, a putative antiepileptic drug, enhances the duration of opening of GABAA receptor channels. Epilepsia 47:704-716.
Ragona E. (2011) Cognitive development in children with Dravet syndrome. Epilepsia 52(Suppl. 2):39-43.

Sakauchi M, Oguni H, Kato I, Osawa M, Hirose S, Kaneko S, Takahashi Y, Takayama R, Fujiwara T. (2011) Retrospective multiinstitutional study of the prevalence of early death in Dravet syndrome. Epilepsia 52:1144-1149.

Skluzacek JV, Watts KP, Parsy O, Wical B, Camfield P. (2011) Dravet syndrome and parent associations: the IDEA League experience with comorbid conditions, mortality, management, adaptation, and grief. Epilepsia 52(Suppl. 2):95-101.

Tanabe T, Awaya Y, Matsuishi T, Iyoda K, Nagai T, Kurihara M, Yamamoto K, Minagawa K, Maekawa K. (2008) Management of and prophylaxis against status epilepticus in children with severe myoclonic epilepsy in infancy (SMEI; Dravet syndrome)-a nationwide questionnaire survey in Japan. Brain Dev 30:629-635.

Thanh TN, Chiron C, Dellatolas G, Rey E, Pons G, Vincent J, Dulac O. (2002) Long-term efficacy and tolerance of stiripentaol in severe myoclonic epilepsy of infancy (Dravet's syndrome). Arch Pediatr 9:1120-1127.

Tran A, Rey E, Pons G, Rousseau M, d'Athis P, Olive G, Mather GG, Bishop FE, Wurden CJ, Labroo R, Trager WF, Kunze KL, Thummel KE, Vincent JC, Gillardin JM, Lepage F, Levy RH. (1997) Influence of stiripentol on cytochrome P450-mediated metabolic pathways in humans: in vitro and in vivo comparison and calculation of in vivo inhibition constants. Clin Pharmacol Ther 62:490-504. 